EPJ Web of Conferences 41, 01012 (2013)

DOI: $10.1051 /$ epjconf/20134101012

(C) Owned by the authors, published by EDP Sciences, 2013

\title{
Spectral Caustics in Attosecond Science
}

\author{
O. Raz, O. Pedatzur, B. D. Bruner and N. Dudovich \\ Department of Physics of Complex Systems, Weizmann Institute of Science, Rehovot 76100 Israel
}

\begin{abstract}
A unique type of singularity common in wave phenomena, known as caustics, links processes observed in many different branches of physics [1]. We investigate the role of caustics in attosecond science and in particular the physical process behind high harmonic generation. By exploiting singularities of the three-step model that describes HHG, we can manipulate and enhance specific features in the emitted harmonic spectrum. This new level of control holds promises in both scientific and technological aspects of attosecond science, and provides a deeper insight into the basic mechanism underlying the high harmonic generation process.
\end{abstract}

\section{Introduction}

Multiple rays of light can coalesce and form bright focusing features which are known as caustics. These features are singularities of ray optics in the sense that their intensity, according to the ray approximation, should diverge. A widely known caustic is the focal point of a lens, which gives infinite intensity according to a simple ray optics calculation. Optical caustics are abundant: from the "cusp" feature on the bottom of a coffee cup to the bright spots of focused light on the bottom of a swimmingpool [1]. Like many other types of singularities, caustics have universal properties. Their geometry, width, enhancement and diffraction patterns can be described in terms of universal classes, according to the dimensionality of the system. Though originating from optics, caustics can be found in many other fields. Gravitational microlensing enhancements that are used to detect extrasolar planets, electron flow focusing in 2D electron gases; and propagation of radio frequencies are all described using the language of caustics. Mathematically, caustics are thoroughly described in terms of catastrophe theory, where each caustic can be associated with a catastrophe class that dictates its properties.

We show that caustics play a major role in the process of high harmonic generation [2]. Caustics offer a deeper understanding of the basic mechanism behind the HHG production, in a regime where the simple semi-classical model describing the production diverges. We experimentally demonstrate a "swallowtail" class caustic accompanied by a dramatic enhancement of a narrow band in the HHG spectrum. The observed enhancement is predicted by catastrophe theory, and cannot be explained by the semiclassical model or by a simple stationary phase approximations of quantum calculations [3]. This approach can be integrated with previously demonstrated enhancement methods, and put towards, for example, the generation of high flux X-rays sources that are attractive for biological and material processing applications.

\section{Experiment}

We experimentally demonstrate a "swallowtail" caustic using a two color excitation field. The fundamental field is produced by a $30 \mathrm{fs}, 1 \mathrm{kHz}, 800 \mathrm{~nm}$ Ti:sapphire laser. The second harmonic field is produced by passing the near infrared field through $\mathrm{a} \mathrm{BaB}_{2} \mathrm{O}_{4}$ crystal, thereby combining the fundamental field with its second harmonic. The ratio of the two fields, $R=\frac{E_{S H}}{E_{I R}}$, and their relative phase $\phi$, are carefully controlled.

This is an Open Access article distributed under the terms of the Creative Commons Attribution License 2.0, which permits unrestricted use, distribution, and reproduction in any medium, provided the original work is properly cited. 

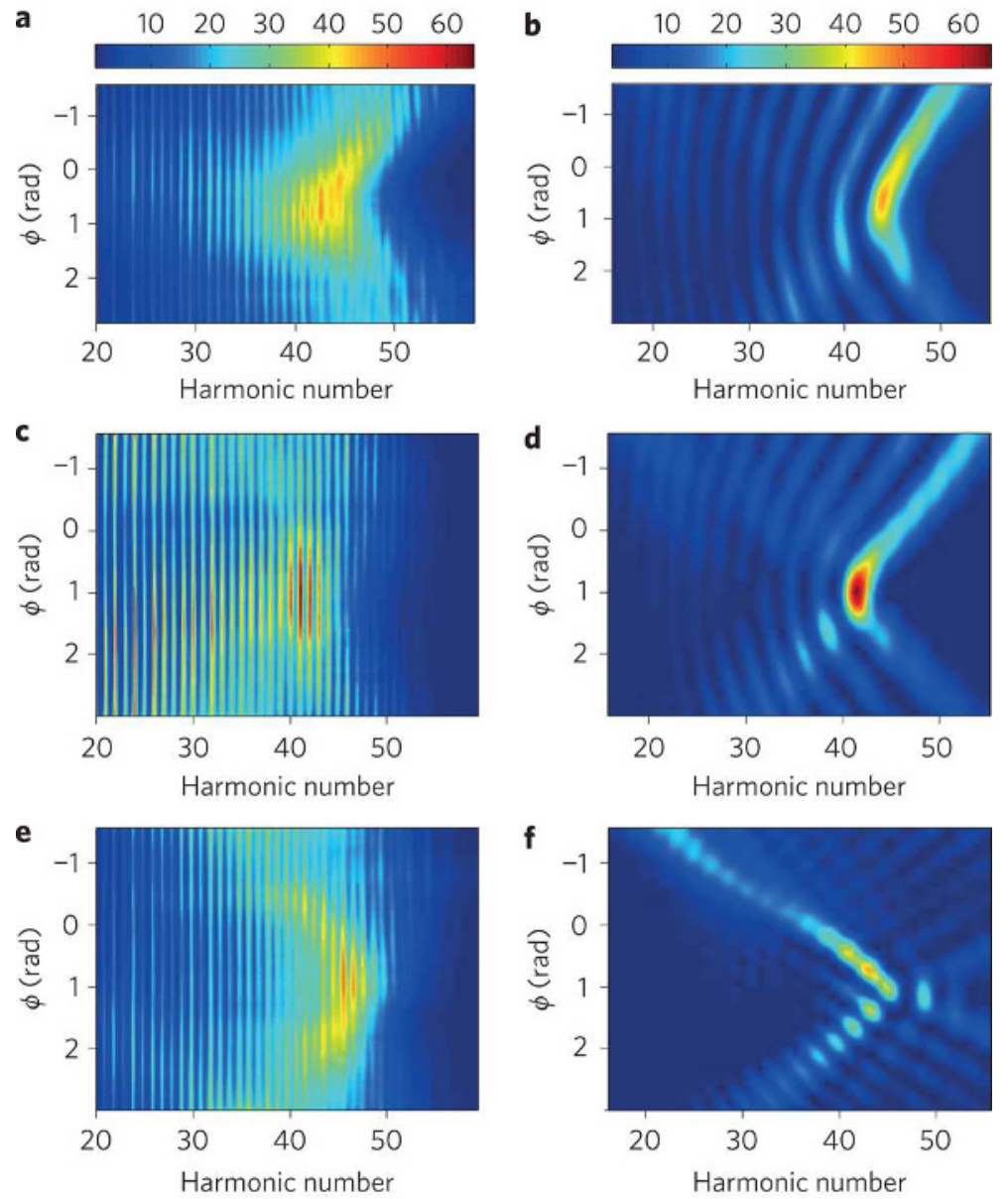

Fig. 1. Demonstration of the swallowtail caustic in HHG. Left-hand column, Figures 1 (a), (c), and (e): experimentally measured HHG spectra as a function of $\phi$ for $R=0.3,0.45$, and 0.75 respectively. Right-hand column, Figures 1 (b), (d), and (f): corresponding theoretical swallowtail diffraction patterns

The swallowtail, like all other structurally stable caustics, is associated with a standard diffraction pattern. By measuring HHG spectra (generated in Ne atoms) while varying the two-colour phase $\phi$, we have measured slices of this diffraction pattern along surfaces of equal $R$. The left column in Figure 1 shows the measured HHG spectral intensity for three different values of $R$. In the right column of Figure 1, the diffraction patterns of the swallowtail are calculated for the corresponding experimental parameters.

\section{Results}

We find an excellent agreement between the expected diffraction pattern and the measured spectra. A systematic pressure scan was performed to verify that propagation effects play no significant role in the observed enhancement. For low values of conversion $R \sim 0.3$, only the enhancement associated with the cutoff singularity is observed in the measured spectra (Figure 1(a)). This enhancement is narrow along the spectral axis but gradually changes along the $\phi$ axis. Its maximum is located at the value of $\phi$ that minimizes the cutoff frequency. A similar response is described in the calculated pattern in Figure 1(b). For larger conversion values $R \sim 0.45$, we observe an extremely sharp enhancement along 
both $\omega$ and $\phi$ at the $40^{\text {th }}$ harmonic, as shown in Figure 1(c). The measured enhancement at the $40^{\text {th }}$ harmonic is of about an order of magnitude with respect to the averaged intensity outside the caustic (low harmonics in Figure 1(a). This narrow feature is well captured by the theoretical diffraction pattern in Figure 1(d). A further increase in the conversion ratio, at $R \sim 0.75$, is shown in Figure 1(e) and Figure 1(f). The change in the curvature describing the location of the cutoff enhancement as a function of f compared to the low R case (Figure 1(a)) is another clear footprint of the swallowtail caustic.

Figure 1(e) demonstrates the spectral controllability induced by the caustic: by changing the relative phase we can scan the dominant harmonics in the spectrum. Moreover, the main features observed in all experiments scale with the total energy, and therefore can be easily tuned. In the measurements, the fine structure of the caustic is partially obscured because of multi-cycle and spatial averaging in our experiment that smear small features. Nevertheless, the main patterns clearly originate from the expected caustic structures.

\section{Conclusions}

The swallowtail in the two color setup is by no means the only case in which caustics appear in HHG, nor is it the only caustic type that can be expected. Other caustics are expected to occur when additional control parameters are introduced, extending the controllability over the spectral intensity.

Caustics offer a simple complementary picture for the semiclassical model that describes HHG. They reveal the quantum nature of the process in a regime where the classical analysis fails. Although our work focuses on the HHG process, caustics are at the heart of a broad range of strong field interactions which have analogous semiclassical models, such as above threshold ionization, electron diffraction or double ionization.

\section{References}

1. M.V. Berry and C. Upstill, in Progress in Optics XVIII (Elsevier 1980, E. Wolf ed.).

2. P. B. Corkum, Phys. Rev. Lett. 71, (1994) 1993-1997.

3. O. Raz, O. Pedatzur, B. D. Bruner and N. Dudovich, Nat. Photonics 6, (2012) 170-173. 